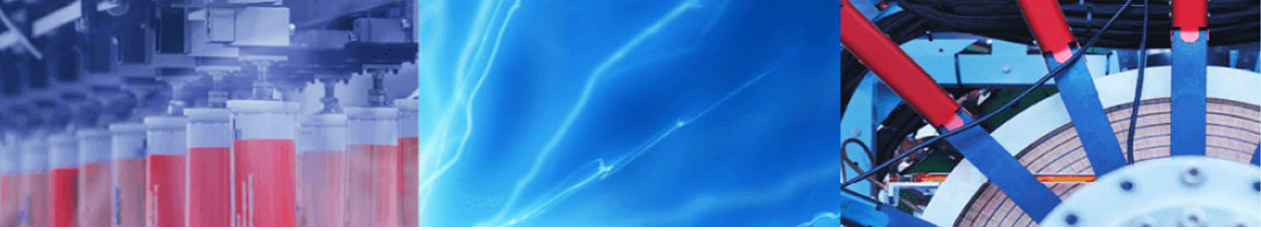

Research Article

\title{
AFM investigation of the influence of ethanol absorption on the surface structure and elasticity of polyamides
}

\author{
Jaime Julio Cervera-Moreno ${ }^{1} \cdot$ Alejandro Martinez-Borquez $^{1} \cdot$ Paul Sotta $^{2} \cdot$ Marco Laurati $^{1}$ D
}

(c) Springer Nature Switzerland AG 2019

\begin{abstract}
We study the variations of surface structure and elasticity of different types of Nylon during absorption of ethanol using AFM surface topography and AFM micro-mechanics. We observe that ethanol absorption induces significant changes in the surface structure, in particular an increase of roughness as well as the presence of local damage. All investigated Nylons soften by a factor 1.5-2.5 as a consequence of ethanol absorption. The Young modulus of the samples as a function of immersion time shows a complex, non-monotonic behavior with a minimum at intermediate immersion times. This behavior results from an interplay between the reduction of the glass transition temperature $T_{g}$, typically observed in bulk, and surface-specific effects such as the onset of surface crystallisation and degradation.
\end{abstract}

Keywords Polyamide AFM - Elasticity · Absorption

\section{Introduction}

Polyamides (PAs), also known as Nylons, are semicrystalline polymers characterized by the presence of amide groups intercalated within linear alkane chains [1-3]. The amide groups form inter-chain hydrogen bonds: different organisations of the hydrogen bond network give rise to different crystalline structures and therefore crystalline polymorphism [4-8]. The hydrogen bond network is affected by the interaction with polar solvents. In semicrystalline polyamides previous studies indicate that absorption of water or ethanol occurs preferentially within the amorphous phase and affects the molecular mobility, resulting in plasticisation as confirmed by NMR [9], dynamic mechanical analysis $[10,11]$ and dielectric spectroscopy measurements [12-16]. The most noticeable plasticization effect, related to an increased molecular mobility, is a drop of the glass transition temperature $T_{g}$ in samples fully saturated with solvent, by about $80 \mathrm{~K}$ for water and up to $100 \mathrm{~K}$ for ethanol. This plasticisation effect has been associated with the polar solvent-induced break up of amide-amide bonds and the formation of more mobile amide-solvent-amide bonds [11]. The solvent induced plasticisation has a significant impact on the macroscopic mechanical properties [1] and on the long term stability of the material, thus affecting durability in applications. In addition there are evidences that solvent absorption is not homogeneous throughout the amorphous phase, due to different degrees of confinement experienced within this phase [17-19]. Most of the solvent is absorbed within the unconstrained, bulk amorphous phase, however a fraction is absorbed into the amorphous phase which is comprised in between the lamellae of the crystalline phase $[12,17$, 20-22], inducing swelling of the lamellar stacking. Moreover, a few experimental studies indicate that absorption of polar solvents could directly influence the morphology of the crystalline phase [12, 22]. Recent studies on the absorption of alcohols, in particular ethanol [22-24], have evidenced the complex nature of this process: it is a non-fickian diffusion process indicating that the diffusion

$\triangle$ Marco Laurati, mlaurati@fisica.ugto.mx | 'División de Ciencias e Ingenierías, Universidad de Guanajuato, Loma del Bosque 103, 37150 León, Mexico. 'Laboratoire Polymères et Matériaux Avancés, UMR5268, CNRS/Solvay Advanced Research and Technology Innovation, 85 Rue des frères Perret, 69192 Saint-Fons Cedex, France. 
coefficient of the solvent is not constant and is affected by the local concentration in the material as well as possibly by the density of hydrogen bonds of the absorbing medium, while the influence of the crystalline phase in not clear yet [24].

The bulk mechanical properties of polyamides and the influence of absorption of polar solvents on them have been previously investigated, indicating Young moduli of the order of a few GPa which suffer a reduction when polar solvents are absorbed into the polymer $[25,26]$. However this drop of modulus at a given temperature is mainly related to the drop in $T_{g}$. In fact, in the glassy regime, it was shown that sorption of water results in an increase of the mechanical rigidity at a given temperature [1]. Most of the studies reported above focus on the bulk properties of the samples and on how they are affected by solvent absorption. However, recent studies suggest the presence of interesting surface structural changes associated with the absorption of ethanol, especially for amorphous polyamides: Preda and coworkers observed the appearance of surface damage in the form of cavities and the increase of crystallinity [24]. In this work we complement this study by determining the surface elasticity of amorphous and semicrystalline polyamides during absorption of ethanol and by linking changes in elasticity to changes in the surface structure. The data have been obtained by Atomic Force Microscopy (AFM). Elasticity was determined by measuring force distance curves, from which the Young modulus of the material was extracted. Surface structure was determined through deflection and topography images. The obtained results provide information which is important for assessing how ethanol absorption affects the durability of nylon parts in industrial applications. Additionally, the impact of absorption on surface properties can have important consequences for materials presenting surface treatments that enhance their barrier properties, obtained through various coating techniques or thin film deposition by e.g. Plasma-Enhanced Chemical Vapor Deposition (PECVD) [27-29]. In particular absorption may have negative consequences on coating adhesion/ cohesion because it affects the polymer surface. The wetting of polymers by solvents that can penetrate into them is also a complex problem which has recently received attention [30]. The approach that we propose in this work may provide information on the changes induced by the solvent on the polymer surface that are complementary to static and dynamic contact angle measurements.

\section{Materials and methods}

In Sect. 2.1 we present the details of sample preparation, in Sect. 2.2 a description of absorption experiments and in Sect. 2.3 the details of the AFM experiments used to determine the topography and elastic properties of the samples' surface.

\subsection{Samples}

Polyamide 6 (PA6), Polyamide 6,6 (PA6,6), Polyamide 6,10 $(\mathrm{PA} 6,10)$ and the aromatic Polyamide 6I (PA6I) were provided by Solvay (France). Figure 1a shows their chemical formulas. The copolymers are formed by condensation of hexamethylenediamine with a diacid: Adipic acid for PA6,6, PA6, 10 and isophthalic acid for PA6I. The degree of crystallinity of the semi-crystalline polymers, PA6, PA6,6 and PA6, 10 was estimated as $\chi_{\mathrm{c}}=\left(\Delta H_{\mathrm{m}}-\Delta H_{\text {rec }}\right) / \Delta H_{\mathrm{m}}^{100 \%}$, where the melting and recrystallization enthalpies, $\Delta H_{\mathrm{m}}$ and $\Delta H_{\text {rec }}$ respectively, were experimentally determined by modulated Differential Scanning Calorimetry (DSC), and $\Delta H_{\mathrm{m}}^{100 \%}$ is the reference enthalpy associated with a $100 \%$ crystalline sample. For PA6, PA6,6 and PA6, 10 we used for $\Delta H_{\mathrm{m}}^{100 \%}$ the value reported in Ref. [31]. We obtained $\chi_{\mathrm{c}}^{6} \approx 38 \%, \chi_{\mathrm{c}}^{6,6} \approx 38 \%$ and $\chi_{\mathrm{c}}^{6,10} \approx 23 \%$. PA6, PA6,6, PA6, 10 and PA6I were provided to us in the form of films with respective thickness: $150 \mu \mathrm{m}, 100 \mu \mathrm{m}, 300 \mu \mathrm{m}$ and $300 \mu \mathrm{m}$. The films of PA6 and PA6,6 were obtained by extrusion using a Leistritz co-rotating twin screw
Fig. 1 Chemical formulas of polyamides, a PA6, b PA6,6, c PA6, 10, d PA6I

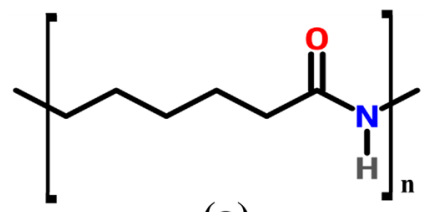

(a)

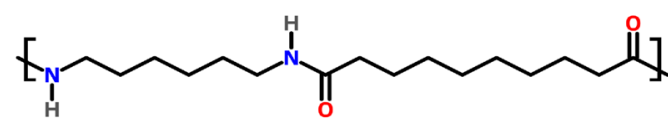

(c)

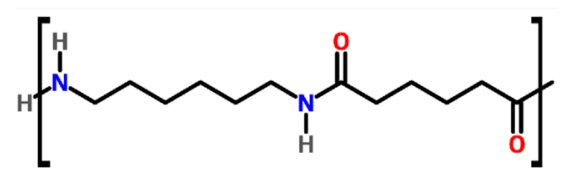

(b)<smiles>CCC(C)C(=O)c1cccc(C(=O)NCCCCCCN(C)F)c1</smiles>

(d) 
extruder (diameter $D=34 \mathrm{~mm}$, ratio $L / D=35$, screw speed $255 \mathrm{rpm}$, throughput $10 \mathrm{~kg} / \mathrm{h}$ and extrusion temperature $557 \mathrm{~K}$ at the die). A planar dye was placed at the end of the extruder and the resulting product was drawn at $2 \mathrm{~m} / \mathrm{min}$ with a draw ratio of 7 through a series of rolls set at $408 \mathrm{~K}$. The amorphous polymer PA6I film were obtained by injection in a Fanuc S-2000i 150A(180T) press (D. Delcourt, Solvay Brussels) in order to obtain injection molded plates of $125 \times 125 \times 1 \mathrm{~mm}$. PA6, 10 pellets were injected in a DEMAG H200-80T press (E. Seignobos, Solvay Belle-Etoile) in order to obtain injection molded plates of $100 \times 100 \times 0.8 \mathrm{~mm}$.

\subsection{Absorption curves}

The absorption of solvent was followed at $T=296 \mathrm{~K}$ by measuring the total mass of the sample as a function of immersion time. The weight at different immersion times was quickly measured after taking out the sample from the immersion medium and after removal of excess solvent from the surface, using a Sartorius ENTRIS64-1S analytical balance with a precision of $0.1 \mathrm{mg}$ and a repeatability of $0.1 \mathrm{mg}$. The sample was re-immersed in the solvent immediately afterwards. Solvent desorption during the weighing procedure could be safely neglected.

\subsection{AFM measurements}

All AFM measurements were performed with an Alpha 300 A microscope (Witec) using a NSG-30 tip (NT-MDT). The tip is made of crystalline $\mathrm{Si}$ and presents the geometry of a vertical cylindrical punch with flat base of length $L=500 \mathrm{~nm}$ and radius of $10 \mathrm{~nm}$. Typical SEM images of the AFM tip can be found in the manufacturer's catalogue [32]. The cantilever constant $k=16 \mathrm{~N} / \mathrm{m}$ was determined by means of the method proposed by Sader [33]. Measurements at different immersion times in ethanol were performed, similar to the absorption curves, after taking out the sample from the immersion medium and after removal of excess solvent from the surface. Given that the measurement of a force distance curve takes approximately $60 \mathrm{~s}$, solvent desorption could be again safely neglected. All measurements were performed at $\mathrm{T}=23^{\circ} \mathrm{C}$.

\subsubsection{Topography}

Topography and deflection images of $40 \times 40 \mu \mathrm{m}^{2}$ were obtained in contact mode by measuring 256 points per line at a velocity of 1 line/s. The velocity was chosen in order to optimize the noise level in the images.

\subsubsection{Force-distance curves}

Force-distance curves were obtained by approaching and indenting the AFM tip into the sample. An approaching speed of $0.5 \mu \mathrm{m} / \mathrm{s}$ was used for all samples. We observed that in the range of speeds $0.5-5.0 \mu \mathrm{m} / \mathrm{s}$ the curves did not depend on the chosen velocity. An indentation range varying from 50 to $150 \mathrm{~nm}$, depending on sample and immersion time, was explored. Within this range, both the linear and non-linear response of the material are explored. In order to extract the Young modulus of the material the initial part of the curves, typically up to an indentation $\delta=5 \mathrm{~nm}$, were modeled with the Harding-Sneddon model for a planar cylindrical punch indenter in contact with an elastic solid [34]. According to this model the Force and indentation $\delta$ are related by the following equation [35]:

$F=\frac{2 R E}{1-v^{2}} \delta$

where $R$ is the cylinder radius, $E$ is the Young's modulus and $v$ is the Poisson's ratio of the material. In this case $R=10 \mathrm{~nm}$ and a value $v=0.39$ was used [1]. A representative curve and corresponding fit of the initial linear response regime according to Eq. 1 are shown in Fig. 2.

\section{Results and discussion}

\subsection{Deflection and topography images}

We characterized the surface morphology of the different investigated polyamides in the original dry state

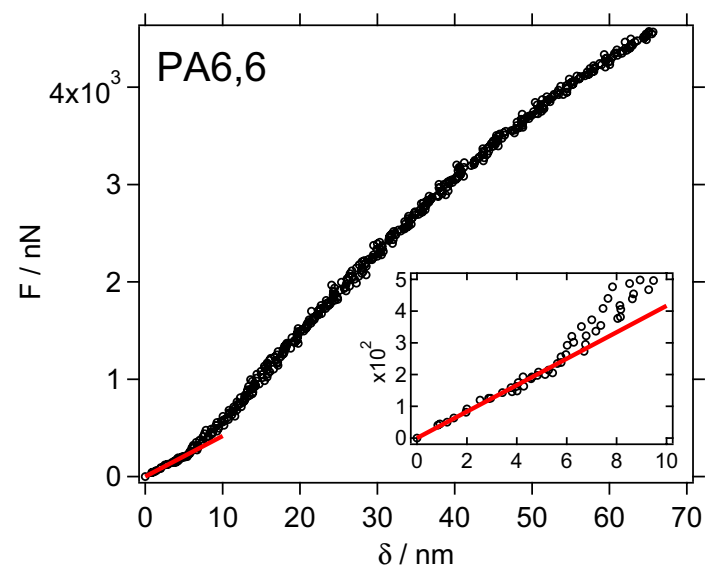

Fig. 2 Representative force-distance curve obtained for dry PA6,6 (symbols) and fit of the initial linear response regime to Eq. 1 (line), describing the force-indentation relation in the Harding-Sneddon model for a planar cylindrical punch indenter. Inset: zoom of the small indentation regime, same quantities and units as in the main plot 
and in the ethanol saturated state using AFM measurements in contact mode, as detailed in Sec. 2.3. We present both deflection (Fig. 3) and topography (Fig. 4) representative images of the samples. In deflection images the voltage associated with the color scale quantifies the degree of positive or negative cantilever deflection compared to the setpoint. Therefore color variations in the deflection images reflect variations in the curvature of the surface along the scan direction. In these images the texture and structural details of the surface are better perceived. In the topography images instead the height variations and changes in roughness can be better quantified. For dry PA6,6 (Figs. 3, 4 top) the typical spherulite structure of semicrystalline polyamide can be clearly seen, with spherulites having sizes of a few $\mu \mathrm{ms}$. In the ethanol saturated state the surface structure appears more diffuse and the spherulites

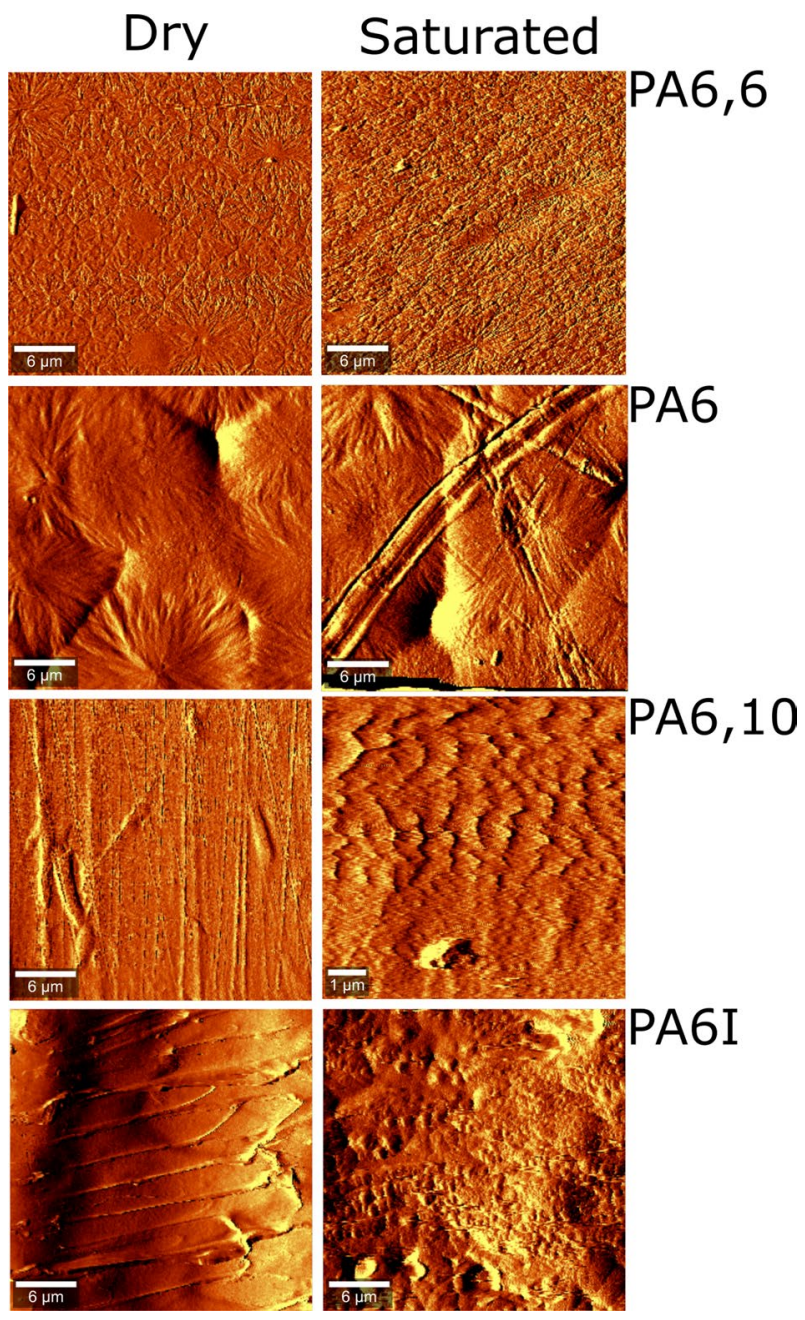

Fig. 3 AFM deflection images of exemplary regions of the samples: PA6,6 (top), PA6 (mid-top), PA6,10 (mid-bottom), PA6I (bottom) in the original dry state (left) and at full saturation of ethanol, corresponding to the longest immersion time of 4 weeks (right) deform and become elongated (Fig. 4). Also in dry PA6 (Figs. 3, 4 mid-top) a clear spherulite structure is present, with the spherulites having a considerably larger size than in PA6,6. In the ethanol saturated sample one can observe a clear deformation of the spherulites, which seems to be associated to a non-homogeneous swelling, as can be best seen in Fig. 3 (top-right). It may be inferred that spherulites are less swollen in the vicinity of the center than in peripheral regions, possibly associated to a gradient of crystallinity fraction going from the center towards the periphery. This phenomenon could be responsible, both in PA6,6 and PA6, for the apparent damage of the surface structure, evidenced by the appearance of cracks (in PA6) and a larger degree of roughness. The dry PA6,10 samples (Figs. 3, 4 mid-bottom) presents a significantly different surface structure compared to PA6,6 and PA6: The surface presents elongated, stretched fiber-like structures instead of spherulites. In the ethanol saturated sample significant changes are observed: The elongated fiberlike structures deform and acquire wavy shapes. Finally, the dry PA6I (Figs. 3, 4 bottom) shows an amorphous structure with some longitudinal cracks on the surface. In the ethanol saturated state we can observe that the longitudinal cracks disappear and are replaced by a number of bumps and some cavities.

We quantified variations in the surface roughness by calculating two commonly used parameters [36], the arithmetic average $R_{a}$ and the mean slope of the profile Rda:

$R a=\frac{1}{n} \sum_{i=1}^{n}\left|y_{i}\right|$

$R d a=\frac{1}{n-1} \sum_{i=1}^{n-1} \frac{\delta_{y_{i}}}{\delta_{x_{i}}}$

where $y_{i}$ is the height of point $i$ with respect to the mean line of the profile, $n$ is the number of points along a line, and $\delta_{y_{i}} / \delta_{x_{i}}$ is the slope between two successive points of the profile. Both quantities were calculated line-by-line and averaged over sub-regions of a surface. We selected sub-regions where no pronounced dips or peaks were present, in order to avoid an artificial bias of the results. Additionally, for each sample results were averaged over different surface images. The obtained parameters are reported in Table 1 for samples in the dry and ethanol saturated states. Both parameters reveal an increase of the surface roughness in the ethanol saturated samples, except for PA6,6, where the average height increases, while the average slope decreases. The decrease of $R d a$ in the ethanol 
Fig. 4 AFM topography images of exemplary regions of the samples: PA6,6 (top), PA6 (midtop), PA6, 10 (mid-bottom), PA6I (bottom) in the original dry state (left) and at full saturation of ethanol, corresponding to the longest immersion time of 4 weeks (right)

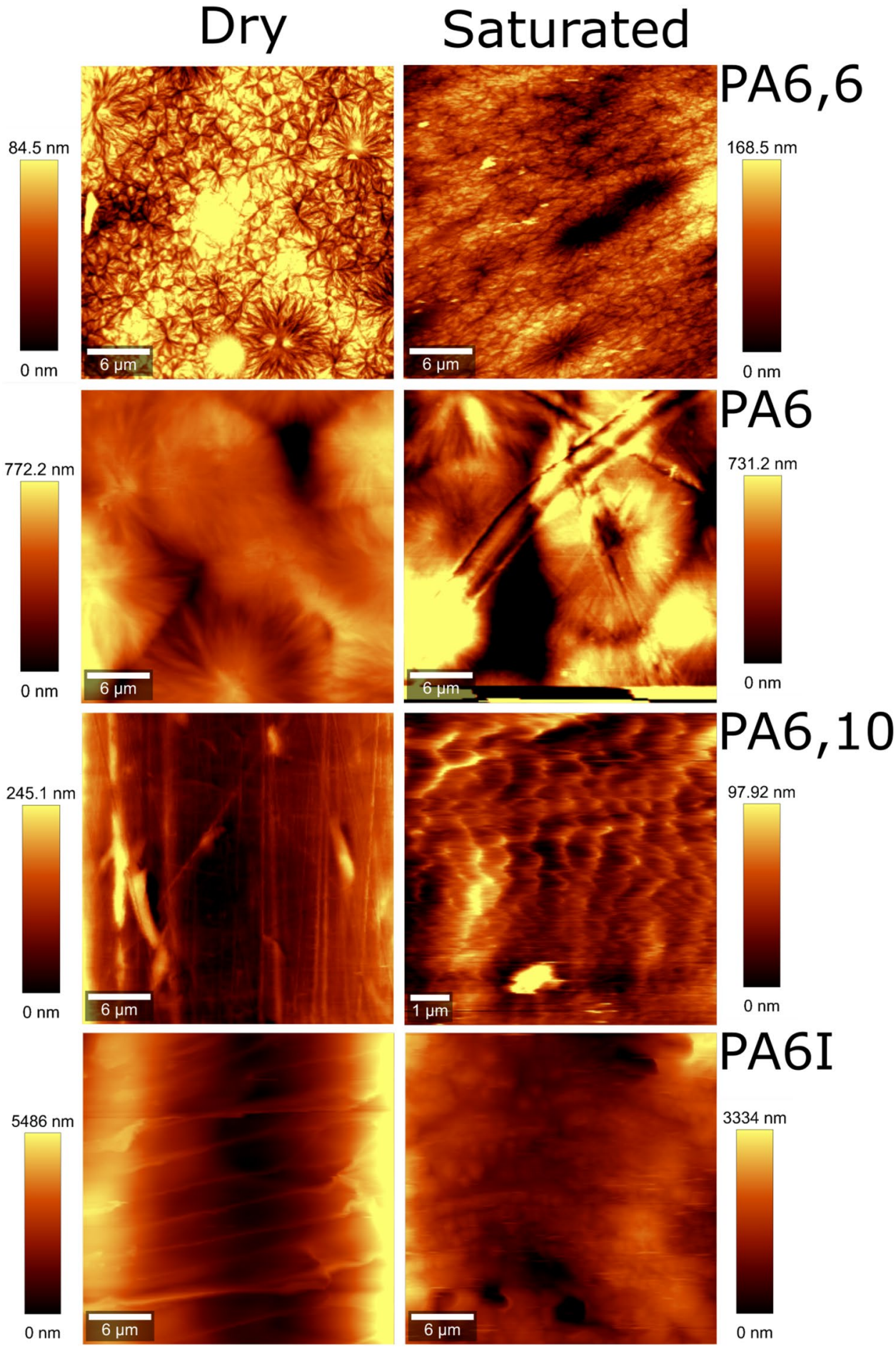

saturated PA6,6 sample might reflect the elongation of the spherulite structures which was discussed earlier and which could lead to an overall smoothing of the surface. We can conclude that for all samples the absorption of Ethanol reveals important modifications of the surface structure, with an increase of surface roughness and a degradation of the structural order, when present.

\subsection{Absorption curves}

We report in Fig. 5 the absorption curves obtained for polyamide PA6,6, PA6, 10, PA6 and PA6I in ethanol. Absorption is quantified through the ratio $\Delta m / \Delta m_{\text {eq }}$, where $\Delta m=m-m_{0}$ is the mass variation with $m$ the mass of the polymer at time $t$ and $m_{0}$ the mass in the initial dry state. The value $\Delta m_{\text {eq }}$ corresponds to the equilibrium value in 
Table 1 Average roughness parameters determined for dry and ethanol saturated (sat) samples: Average height $R a$ and Average slope $R d a$

\begin{tabular}{llr}
\hline Sample & Ra $(\mu \mathrm{m})$ & Rda \\
\hline PA6 dry & 3.8 & 40 \\
PA6 sat & 92 & 204 \\
PA6,6 dry & 1.0 & 68 \\
PA6,6 sat & 7.8 & 43 \\
PA6,10 dry & 0.2 & 21 \\
PA6,10 sat & 0.8 & 40 \\
PA6I dry & 5.1 & 58 \\
PA6I sat & 6.5 & 77 \\
\hline
\end{tabular}

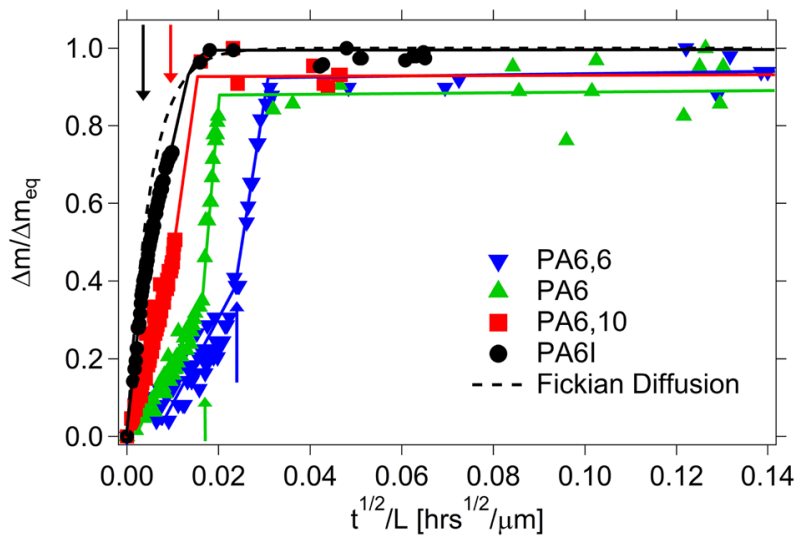

Fig. 5 Absorption curves for different polyamides in ethanol, as indicated, showing the relative increase of the samples mass with respect to the initial mass of the dry sample $\Delta m=m-m_{0}$, with $m_{0}$ the mass of the dry sample, normalized by the equilibrium value at long immersion times $\Delta m_{\text {eq }}$ as a function of $\sqrt{t} / L$, with $L$ the sample thickness. Lines are guides for the eye. Arrows indicate the position where a change of slope with respect to the initial Fickian diffusion is observed for the different samples. The dashed line indicates a representative Fickian diffusion curve with constant diffusion coefficient corresponding to the initial slope of PA6I

the saturated sample, at long immersion times. The ratio $\Delta m / \Delta m_{\text {eq }}$ is plotted against $\sqrt{t} / L$, with $L$ the sample thickness, to highlight Fickian diffusion, which corresponds to a linear dependence of $\Delta m / \Delta m_{\text {eq }}$ on $\sqrt{t} / L$. This is observed in the initial regime of the sorption curves. Normalization by $L$ takes into account the effect of the sample thickness. Overall, all absorption curves show non-fickian behavior, which is typically observed when the solvent diffusion time is comparable to the polymer relaxation time and/or when the mobility of the polymer changes during sorption (due to plasticization). Note that both statements or hypothesis are closely related, even though not fully equivalent. Indeed, the polymer may be plasticized if its relaxation time becomes comparable to the solvent diffusion time along the sorption curve. This competition of timescales gives rise, close to the polymer surface, to a region in which the polymer is swollen. This region slowly expands within the sample and the solvent penetrates more rapidly within the remaining dry material. The response is different for the amorphous and semicrystalline polymers. The amorphous sample PA6I shows an initial (diffusive) regime of linear mass increase, followed by a non-linear regime in which the mass increases sublinearly until saturation is reached. Note that, as shown in Fig. 5, the deviation from Fickian diffusion, characterized by a constant diffusion coefficient, is moderate. We can estimate a diffusion coefficient $D$ of order $10^{-12} \mathrm{~m}^{2} / \mathrm{s}$ for PA6I. For this polymer, previous studies showed that the change of slope of the absorption curve coincides with the appearance of surface damage in the form of cavities and crystallization [24]. The relative mass increase is about $16 \%$. Both the shape of the absorption curve and the relative mass increase are in agreement with previous studies [24]. The semicrystalline polymers PA6 and PA6,6 present similar absorption curves, with an initial Fickian regime presenting an absorption rate slower than for PA6I, as indicated by the smaller slope. Slower diffusion is expected as a result of the tortuosity induced by the crystalline structure. The Fickian regime presents a slower absorption rate for PA6,6 than for PA6, which might be associated with the more compact crystalline spherulite structure indicated by the AFM images (Fig. 3). For longer times a second Fickian regime is observed in a narrow interval of times and presents a significantly larger absorption rate. Finally, at the longest times saturation is achieved. Previous studies showed that while surface damage is less important than in amorphous PA, changes in the crystallinity degree and morphology are observed during the second Fickian regime [24]. The relative mass increase is about $9 \%$ for PA6,6 and about $11 \%$ for PA6, eventually indicating a larger degree of crystallinity for PA6.6. The shape of the curves and the steady state mass increase are also in agreement with previous studies [24]. Finally, the shape of the curves and the equilibrium mass increase for PA6, 10 are also similar to PA6 and PA6,6, however the absorption process is faster, as indicated by the larger initial slope compared to PA6 and PA6,6. The relative mass increase is about $9 \%$.

\subsection{Elasticity during absorption}

The average surface elasticity of the PA samples was determined by measuring force-indentation curves. The curves were first measured for the dry samples and successively for different immersion times in ethanol. We report in Fig. 6 representative force-indentation curves obtained for PA6 at different immersion times. Depending on immersion time, one can observe significant variations in the force needed to achieve a determined indentation; additionally these variations do not show a monotonic trend with increasing the immersion time. We will come back to this 


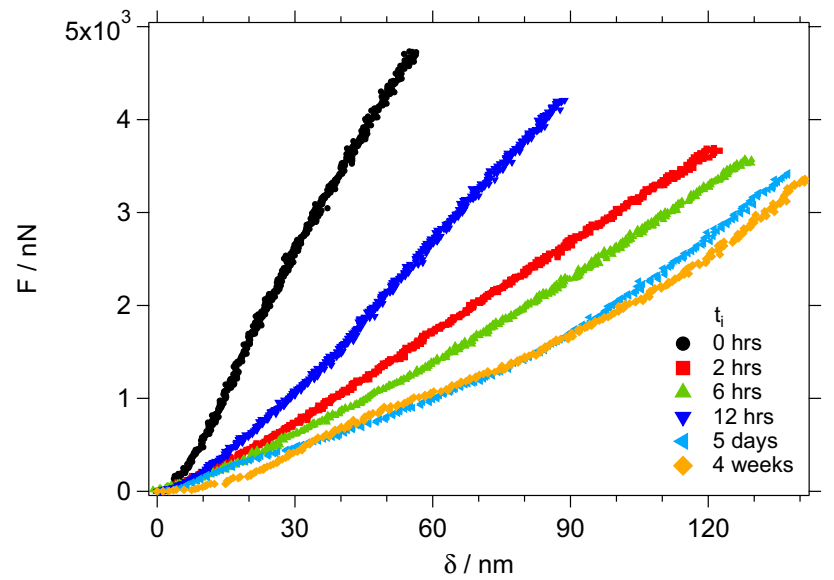

Fig. 6 Force-indentation curves measured for PA6I at different immersion times $t_{i}$ in ethanol, as indicated

point in more detail later. All different PA samples show similar average responses. The initial part of the curves, corresponding to the linear response regime, was fitted using the Harding-Sneddon model for a flat cylindrical punch indenter (Eq. 1) to obtain the Young's modulus $E$ for different immersion times. For the dry samples we obtained the values reported in Table 2: We found $1.9 \mathrm{GPa}<E<3.5 \mathrm{GPa}$, depending on the sample. These values are in good agreement with those found in the literature using rheology or AFM [1].

The Young's moduli obtained as a function of immersion time indicate a reduction of the material's elasticity associated with ethanol absorption (Fig. 7). For all samples the value of $E$ in the regime of solvent saturation is a factor between 1.5 and 2.5 smaller than in the dry material (Fig. 7, inset). The reduction of $E$ can be interpreted as arising from the plasticization of the material induced by the presence of ethanol. The ethanol molecules primarily penetrate the amorphous regions of the material, breaking up the hydrogen bonds between amide groups and intercalating between them. The new hydrogen bonds in which ethanol intercalates between amide groups are more flexible leading to enhanced dynamics of the amid groups, as demonstrated through broadband dielectric spectroscopy [24]. As noted above, the decrease of $E$ is mostly due to the decrease in $T_{g}$. While the measurement temperature (room temperature) is well below $T_{g}$ in the dry state, it is much

Table 2 Young's modulus $E$ of dry PAs obtained by fitting the initial part of force-indentation curves using Eq. 1

\begin{tabular}{ll}
\hline Sample & E (GPa) \\
\hline PA6 & $2.22 \pm 0.03$ \\
PA6,6 & $2.54 \pm 0.02$ \\
PA6,10 & $1.99 \pm 0.03$ \\
PA6I & $3.49 \pm 0.33$
\end{tabular}

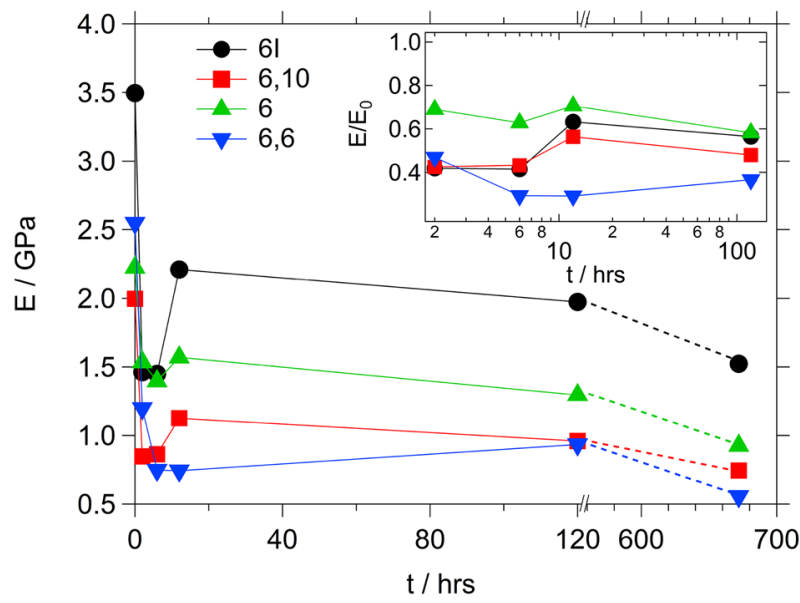

Fig. 7 Young modulus $E$ as a function of immersion time $t$ in ethanol, for all PA samples (as indicated). The bottom axis was split in order to make the initial points better visible. Inset: $E / E_{0}$, being $E_{0}$ the Young modulus in the dry state, as a function of immersion time, limited to the interval $2 \mathrm{~h}<t<120 \mathrm{~h}$ to better evidence the non-monotonic variation of $E / E_{0}$ at short immersion times

closer to or even in the close vicinity of $T_{g}$ in the saturated case, hence the large decrease of modulus. The relative reduction of $E$ compared to the value in the dry state $\left(E_{0}\right)$ is smallest for PA6, largest for PA6,6 and intermediate for $P A 6 I$ and PA6, 10. The values are in any case comparable. The decay of $E$ as a function of immersion time is, however, non-monotonic: After an initial strong decrease of the modulus within the first $1-6 \mathrm{~h}$, all PA show a recovery of $E$ typically around $10 \mathrm{~h}$ of immersion ( $100 \mathrm{~h}$ for PA6,6, inset of Fig. 7) before decreasing again slowly until the saturation value is achieved (Fig. 7).

Comparing with the absorption data, we should first notice that according to our estimate of the diffusion coefficient of ethanol in PA6I, the diffusion time over the distance probed by AFM (of order $150 \mathrm{~nm}$ ) is approximately $1 \mathrm{~ms}$. The variations of elasticity that we observed are on the timescale of hour, which means that they are associated to slow structural evolution of the material in the presence of solvent, in agreement with previous work [22]. Additionally, we determined that the region of the minimum of $E$ roughly corresponds to the region where a change of slope is observed in the absorption curves, while the final slow decrease roughly corresponds to the saturated regime. We recall that, for both amorphous and semicrystalline PAs, the change of slope is associated with variations of crystallinity. Thus, one can speculate that the initial fast reduction of elasticity is associated with the initial softening due to the ethanol absorbed within the amorphous phase of the PA. When the solvent absorption starts to induce crystallisation, an increase of elasticity is observed up to the saturated regime, where the 
crystallisation typically slows down. The final decay would be then associated with further plasticisation of the material and/or it might be related to the degradation of the surface structure evidenced by the AFM measurements of the ethanol saturated samples.

\section{Conclusions}

The surface structure and elasticity of one amorphous and three different semi-crystalline polyamides were determined by AFM in the dry state and as a function of ethanol absorption. For all samples we observed that absorption of ethanol induced significant changes in the surface structure, in particular an increase of the surface roughness and the onset of damage. In the semicrystalline materials surface damage seems to be associated with non-uniform swelling of spherulites and the presence of a gradient of crystallinity. Independently of their internal structure, we found that absorption of ethanol induced a progressive reduction of the Young's modulus, which was found to be about a factor 1.5-2.5 (depending on polymer) smaller in the fully saturated state compared to the dry state. The initial reduction of the Young's modulus can be associated to the enhanced mobility of the amorphous phase induced by the presence of ethanol and the replacement of amide-amide hydrogen bonds with amide-ethanol-amide bonds. After this initial regime, the Young's modulus showed a non-monotonic evolution for absorption times in between 1 and $10 \mathrm{~h}$, with a partial recovery of elasticity at intermediate absorption times which can be related to the onset of crystallisation (amorphous PA) or to changes in the crystallinity degree (semicrystalline PA). A final softening is thought to correspond to slow rearrangements of the hydrogen bond network in the fully wet material and it might also depend on the degradation of the surface structure observed in the deflection and topography images of ethanol saturated samples. Our results show that variations in surface elasticity cannot be only explained through the solvent-induced reduction of $T_{g}$ observed in bulk. Additional surface-specific effects, like changes in crystallinity and surface damage need to be considered. We expect our results to provide useful information to assess the durability of nylon parts in industrial applications in which these plastics are in direct contact with ethanol (or other polar solvents). They might also contribute to the understanding of coating degradation and adsorption in materials where the solvent additionally penetrates into the bulk.

Acknowledgements JJCM, AMB and ML acknowledge support from Conacyt through Grant No. LANIMFE-279887-2017.

\section{Compliance with ethical standards}

Conflict of interest The authors declare that they have no conflict of interest.

\section{References}

1. Kohan MI (ed) (1995) Nylon plastics handbook. Hanser, New York

2. Aharoni SM (1997) n-Nylons: their synthesis, structure, and properties. Wiley, New York

3. Reimschuessel HK (1985) Nylon 6. Chemistry and mechanisms. J Polym Sci Part D Macromol Rev 12:1977

4. Magill JH (1966) Formation of spherulites in polyamide melts. 3. Even-even polyamides. J Polym Sci Part A 2 Polym Phys 4:243

5. Khoury $F(1958)$ The formation of negatively birefringent spherulites in polyhexamethylene adipamide (Nylon 66). J Polym Sci 33:389-403

6. Mann J, Roldan-Gonzalez L (1962) Orientation in nylon spherulites: study by X-ray diffraction. J Polym Sci 60:1

7. Lovinger AJ (1978) Crystallographic factors affecting structure of polymeric spherulites. 1. Morphology of directionally solidified polyamides. J Appl Phys 49:5003-5013

8. Lovinger AJ (1978) Crystallographic factors affecting structure of polymeric spherulites. 2. X-ray-diffraction analysis of directionally solidified polyamides and general conclusions. J Appl Phys 49:5014-5028

9. Miura H, Hirschinger J, English AD (1990) Segmental dynamics in the amorphous phase of nylon 66: solid state deuterium NMR. Macromolecules 23:2169

10. McCrum NG, Read BE, Williams G (1967) Anelastic and dielectric effects in polymer solids. Wiley, New York

11. Starkweather HW (1980) Water in polymers. ACS Symposium Series, vol 127. American Chemical Society, Washington, DC

12. Murthy NS, Stamm M, Sibilia JP, Krimm S (1989) Structural changes accompanying hydration in nylon 6 . Macromolecules 22:1261

13. Laredo E, Hernandez MC (1997) Moisture effect on the low-and high-temperature dielectric relaxations in nylon-6. J Polym Sci Part B Polym Phys 35:2879

14. Pathmanathan K, Cavaillé JY, Johari GP (1992) The dielectric properties of dry and water-saturated nylon-12. J Polym Sci Part B Polym Phys 30:341

15. Neagu RM, Neagu E, Kyritsis A, Pissis P (2000) Dielectric studies of dipolar relaxation processes in nylon 11. J Phys D Appl Phys 33:1921

16. Le Huy HM, Rault J (1994) Remarks on the $\alpha$ and $\beta$ transitions in swollen polyamides. Polymer 35:136-139

17. Pleštil J, Baldrian J, Ostanevich YM, Bezzabotnov VY (1991) Nylon single crystals. J Polym Sci 44:449-458

18. Kolesov I, Androsch R (2012) The rigid amorphous fraction of cold-cristallized polyamide 6. Polymer 53:4770-4777

19. Chen $H$, Cebe $P(2007)$ Investigation of the rigid amorphous fraction in Nylon-6. J Therm Anal Calorim 89:417-425

20. Murthy NS, Akkapeddi MK, Orts WJ (1998) Analysis of lamellar structure in semicrystalline polymers by studying the absorption of water and ethylene glycol in nylons using small-angle neutron scattering. Macromolecules 31:142

21. Murthy NS, Wang ZG, Hsiao BS (1999) Interactions between crystalline and amorphous domains in semicrystalline polymers: small-angle X-ray scattering studies of the Brill transition in nylon 6,6. Macromolecules 32:5594-5599 
22. Laurati M, Arbe A, de Anda AR, Fillot LA, Sotta P (2014) Effect of polar solvents on the crystalline phase of polyamides. Polymer 55(12):2867. https://doi.org/10.1016/j.polymer.2014.04.031

23. Anda ARD, Fillot L, Preda F, Rossi S, Long D, Sotta P (2014) Sorption and plasticization effects of ethanol-toluene-isooctane ternary mixtures in polyamide 6,6 and induced plasticization effects. Eur Polym J 55:199. https://doi.org/10.1016/j.eurpo lymj.2014.04.001

24. Preda F (2016) Dynamics of polyamide in the solid state in presence of solvents and in the molten state. Ph.D. thesis, Université de Lyon

25. Harper C (2000) Modern plastics handbook. McGraw-Hill handbooks. McGraw-Hill, New York

26. Jee AY, Lee M (2010) Comparative analysis on the nanoindentation of polymers using atomic force microscopy. Polym Test 29(1):95. https://doi.org/10.1016/j.polymertesting.2009.09.009

27. Walther M, Heming M, Spallek M (1996) Multilayer barrier coating system produced by plasma-impulse chemical vapor deposition (PICVD). Surf Coat Technol 80:200

28. Garbassi F, Occhiello E (1999) Plasma deposition of silicon-containing layers on polymer substrates. Macromol Symp 139:107

29. Lange J, Weyser Y (2003) Recent innovations in barrier technologies for plastic packaging: a review. Packag Technol Sci 16:149
30. Lequeux F, Talini L, Verneuil E, Delannoy G, Valois P (2016) Wetting of polymers by their solvents. Eur Phys J E 39, 12

31. Lim LT, Britt IJ, Tung MA (1999) Sorption and transport of water vapor in nylon 6,6 film. J Appl Polym Sci 71:197

32. NT-MDT Spectrum Instruments, AFM Probes and Accessories Catalogue (2019). https://www.ntmdt-tips.com/data/media/ nt-mdt-si_afm_probes.pdf. Accessed 30 Sept 2019

33. Sader JE, Chon JWM, Mulvaney P (1999) Calibration of rectangular atomic force microscope cantilevers. Rev Sci Instrum 70(10):3967. https://doi.org/10.1063/1.1150021

34. Harding JW, Sneddon IN (1945) The elastic stresses produced by the indentation of the plane surface of a semi-infinite elastic solid by a rigid punch. Proc Camb Philos Soc 41(1):16. https:// doi.org/10.1017/S0305004100022325

35. Johnson KL (1985) Contact mechanics. Cambridge University Press, Cambridge. https://doi.org/10.1017/CBO9781139171731

36. Gadelmawla ES, Koura MM, Maksoud TMA, Elewa IM, Soliman HH (2002) Roughness parameters. J Mater Proc Technol 123:133

Publisher's Note Springer Nature remains neutral with regard to jurisdictional claims in published maps and institutional affiliations. 